\title{
Nanorobotics for Electron and Ion Microscopy
}

\author{
Volker Klocke
}

Klocke Nanotechnik, Pascalstr. 17, 52076 Aachen, Germany, info@nanomotor.de

The demand for precise in-situ manipulation and handling inside of electron and ion microscopes increased rapidly in the last time. But these microscopes have a wide amplification range and actuators should fit in range and resolution. Therefore an easy to use manipulator has to fulfill a lot of demands, like cartesian movement of independent axes, absolute positioning, high stroke at resolution of a single Nanometer, crash resistance, modular design and many options to fix tools easily. Klocke Nanotechnik developed a Nanorobotics series that fulfills these demands and includes an extensive set of accessory.

In this presentation the technology of Nanorobotics stages, manipulators, microgrippers and other tools will be described as introduction. The main part will be a review about different applications of research centers around the world. Nanorobotics extend electron and ion microscopes from an analytical instrument to a material processing system. Examples described in detail are systems for wafer probing, TEM lamella handling in Focused Ion Beam chambers and Nanomanipulation of small particles like carbon nanotubes, magnetic beads, or samples from life science and forensics.

The Nanorobotics series builds a bridge between nanotechnology and the classical mechanical engineering. It combines the advantages of both technologies: the backlash free movement with resolution better one Nanometer and the capability of kilograms of load at Centimeters stroke. This series of the smallest and most precise positioning modules offers plenty degrees of freedom in smallest volume and fits into any microscope chamber. Integrated position sensors allow the automatic usage with a repeatability better $60 \mathrm{~nm}$. The Nanorobotics series include a set of linear stages with strokes between 5 and $50 \mathrm{~mm}$, microgrippers, sensors, "Nanofinger", nano-indenter and Scanning Probe systems. The following pictures show examples of Nanorobotics modules: 


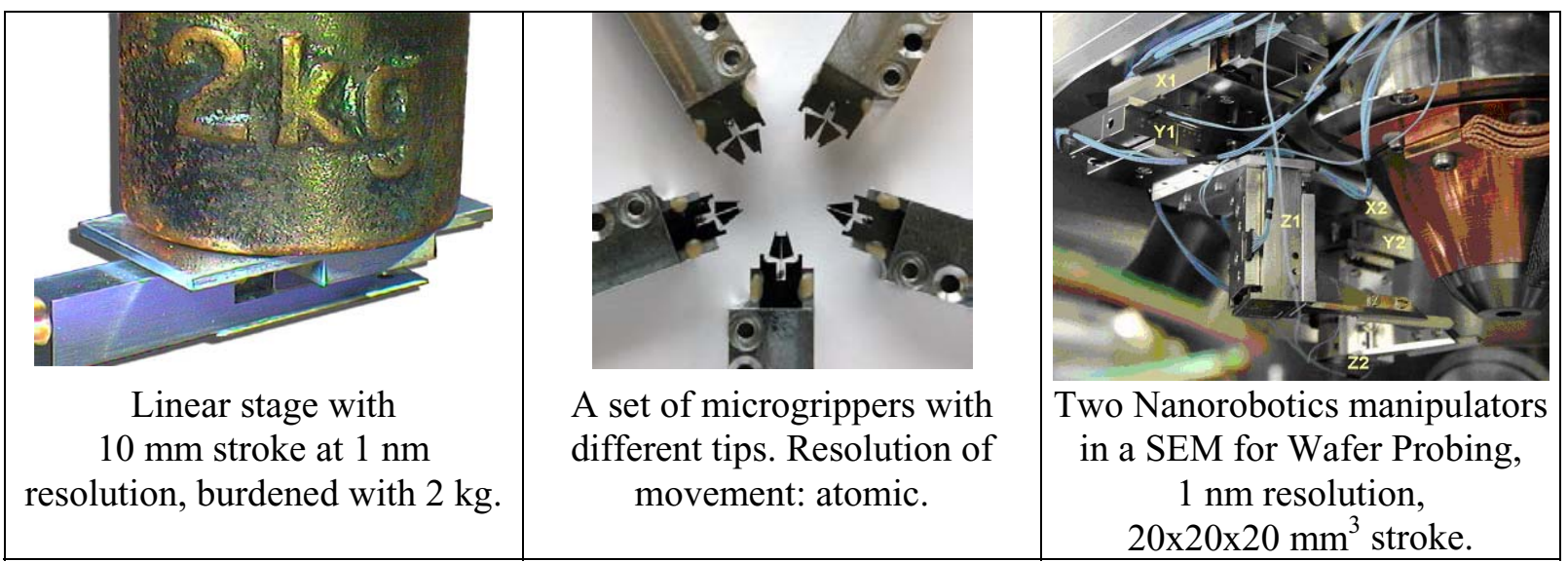

The following examples are an extract from the applications that will be described.

Wafer Probing: The actual small wafer structures cannot be observed in a light microscope anymore. Only two instruments can be used for this task: the AFM expanded to a Wafer Prober or the SEM equipped with Wafer Probers. The AFM cannot resolve structures that are only 2dimensional and ends at a maximum scanning range of about $50 \times 50 \mathrm{um}^{2}$. It can take a lot of time to find a certain sample position with this slow device. The Electron Microscope can zoom from $\mathrm{cm}^{2}$ sized areas into a few $10 \mathrm{~nm}^{2}$ sized areas within a few seconds. And any kind of structures are visible in a SEM, independent if they are flat or not. Therefore Wafer Probing in a SEM is the best solution. A high-end SEM is not necessary, any (elder) SEM can be used for this application.

XRay Imaging: A special SEM including Nanorobotics provides absorption and phase contrast Xray images with resolution better than $120 \mathrm{~nm}$ to reveal fine internal structures and edge details. This technology offers images showing internal sample structures, micro-tomography and stereo images without effort for sample preparation.

TEM lamella preparation in a FIB chamber: Electronic dropouts in integrated circuits that result from structural defects can be inspected by high-resolution transmission electron microscopy measurements. This requires to cut a very small lamella out of the dropout-area. Using ultra precise manipulators renders the complicated preparation techniques much easier. Automatic tip approach and even pattern recognition tools allow a fast industrial application. 